\title{
Initiation of HIV Therapy
}

\author{
Charlotte Yuk-Fan Ho \\ Telephone: +44 (0)20 78825555 ext. 4777 Fax: +44 (0)20 78827997 Email: c.ho@qmul.ac.uk
}

School of Mathematical Sciences, Queen Mary, University of London, Mile End Road, London, E1 4NS, United Kingdom.

*Bingo Wing-Kuen Ling

Telephone: 44 (0)20 78482294 Fax: 44 (0)20 78482932 Email: wing-kuen.ling@kcl.ac.uk

Department of Electronic Engineering, Division of Engineering, King's College London, Strand, London, WC2R 2LS,

United Kingdom.

\begin{abstract}
In this paper, we numerically show that the dynamics of the HIV system is sensitive to both the initial condition and the system parameters. These phenomena imply that the system is chaotic and exhibits a bifurcation behavior. To control the system, we propose to initiate an HIV therapy based on both the concentration of the HIV-1 viral load and the ratio of the CD4 lymphocyte population to the CD8 lymphocyte population. If the concentration of the HIV-1 viral load is higher than a threshold, then the first type of therapy will be applied. If the concentration of the HIV-1 viral load is lower than or equal to the threshold and the ratio of the CD4 lymphocyte population to the CD8 lymphocyte population is greater than another threshold, then the second type of therapy will be applied. Otherwise, no therapy will be applied. The advantages of the proposed control strategy are that the therapy can be stopped under certain conditions, while the state variables of the overall system is asymptotically stable with fast convergent rate, the concentration of the controlled HIV-1 viral load is monotonic decreasing, as well as the positivity constraint of the system states and that of the dose concentration is guaranteed to be satisfied. Computer numerical simulation results are presented for an illustration.
\end{abstract}

Keywords: Initiation of HIV therapy, switching control strategy, chaos control, bifurcation. 


\section{INTRODUCTION}

In 2005, there were about 38.6 millions of adults and children suffering from the human immunodeficiency virus (HIV). The total infected population is increasing year by year [8]. There are about twenty medications approved by the Food and Drug Administration (FDA) of the US government. These medications can suppress the acquired immune deficiency syndrome (AIDS).

It was reported in [9]-[27] that the response of the CD4 lymphocyte population, the CD8 lymphocyte population and the HIV-1 viral load can be characterized by a first order nonlinear differential equation. In this paper, we numerically show that the system dynamics is sensitive to both the initial condition and the system parameters. This implies that the system is chaotic and exhibits a bifurcation behavior. Moreover, the concentrations of the CD4 lymphocyte population, the CD8 lymphocyte population, the HIV-1 viral load and the dose are required to be non-negative and bounded, this implies that the positivity constraint of the system states and that of the dose concentration is required to be satisfied [5]. Because of the chaotic nature of the system and the positivity constraint, the system is very difficult to be controlled. Furthermore, the system has two equilibrium points and one of them is unstable [1]. This implies that a very small derivation of the system vectors from the equilibrium point would cause the system trajectory to diverge locally. In order to control the concentration of the HIV-1 viral load being exactly equal to zero within a certain period of time, zero boundary conditions of the controlled system are required to be satisfied. However, it was reported in [7] that the corresponding linearized system (linearized at the unstable equilibrium point) is uncontrollable, so linear control methods would usually result to the loss of control near the equilibrium point.

The simplest existing strategy for controlling the HIV-1 viral load is via the P control law [1], [2], [4], that is the dose concentration is directly proportional to the concentration of the HIV-1 viral load. However, this control strategy fails to satisfy the positivity constraint and in general this control method does not guarantee the asymptotical stability of the system. Optimal control methods [3], [6] are also proposed. However, all these control methods are derived based on the corresponding 
linearized model, in which the system will lose control when the trajectory is close to the equilibrium point [7].

As the medications have strong side effects, medical professions would prefer to initiate the therapy only at certain situations [7]. However, there is no standard rule for the determination of the time instant that the therapy initiates. Also, the corresponding dose concentration is unknown. In this paper, we propose to initiate the therapy based on both the concentration of the HIV-1 viral load and the ratio of the $\mathrm{CD} 4$ lymphocyte population to the $\mathrm{CD} 8$ lymphocyte population. If the concentration of the HIV-1 viral load is higher than a threshold, then the first type of therapy will be applied. If the concentration of the HIV-1 viral load is lower than or equal to the threshold and the ratio of the CD4 lymphocyte population to the CD8 lymphocyte population is greater than another threshold, then the second type of therapy will be applied. Otherwise, no therapy will be applied. The advantages of the proposed control strategy are that the therapy can be stopped under certain conditions, while the state variables of the overall system is asymptotically stable with fast convergent rate, the concentration of the controlled HIV-1 viral load is monotonic decreasing, as well as the positivity constraint of the system states and that of the dose concentration is guaranteed to be satisfied.

The outline of this paper is as follows: The HIV model is reviewed in Section II. In Section III, the sensitivity of the initial condition and the system parameters against the system dynamics is simulated. The obtained results imply that the system is chaotic and exhibits a bifurcation behavior. In Section IV, a control strategy is proposed. Computer numerical simulation results are also presented. Finally, a conclusion is drawn in Section V.

\section{HIV MODEL}

Denote $a, b, c, d, e$ and $f$ as the system parameters, in which all are positive. Denote $x(t)$, $y(t)$ and $z(t)$ as the concentrations of the CD4 lymphocyte population, the CD8 lymphocyte population and the HIV-1 viral load, respectively. Denote $x_{0}$ and $y_{0}$ as the normal unperturbed 
concentrations of the CD4 and CD8 lymphocyte population, respectively, in which they are also positive. The response of the concentrations of the CD4 lymphocyte population, the CD8 lymphocyte population and the HIV-1 viral load can be characterized by the following first order nonlinear differential equation [9]-[27]:

$$
\begin{aligned}
& \dot{x}(t)=a\left(x_{0}-x(t)\right)-b x(t) z(t), \\
& \dot{y}(t)=c\left(y_{0}-y(t)\right)+d y(t) z(t)
\end{aligned}
$$

and

$$
\dot{z}(t)=z(t)(e x(t)-f y(t)) .
$$

It can be checked easily that the equilibrium points of the system are

$$
\left[\begin{array}{lll}
x_{0} & y_{0} & 0
\end{array}\right]^{T}
$$

and

$$
\left[\begin{array}{lll}
\frac{a e d x_{0}+b f c y_{0}}{e(a d+b c)} & \frac{a e d x_{0}+b f c y_{0}}{f(a d+b c)} & \frac{a c\left(e x_{0}-f y_{0}\right)}{a e d x_{0}+b f c y_{0}}
\end{array}\right]^{T}
$$

\section{SENSITIVITY OF INITIAL CONDITION AND SYSTEM PARAMETERS AGAINST SYSTEM DYNAMICS}

Figure 1a shows the state responses of the system, while Figure 1b shows the corresponding state trajectory, in which $a=0.25, b=50, c=0.25, d=10, e=0.01, f=0.006, x_{0}=1000$, $y_{0}=550, x(0)=x_{0}, y(0)=y_{0}$ and $z(0)=0.03$. It can be seen from Figure $1 \mathrm{~b}$ that the system exhibits a complex behavior. Figure 2 plots the Lyapunov exponents of the system at different time instants with the system parameters being the same as that applied in Figure 1 and with the initial condition being the state vectors at the corresponding time instants. It can be seen from Figure 2 that the Lyapunov exponent of $z(t)$ is positive during the transient moment and at $t=4.15$ year. Figure 3 plots the difference of the state responses between the following two sets of initial conditions with the same set of system parameters applied in Figure 1: $x(0)=442, y(0)=736$ and $z(0)=0.0063$, and its 
$1 \%$ increment, that is $x(0)=446.42, y(0)=743.36$ and $z(0)=0.0064$. It can be seen from Figure 3 that the difference of the state responses between the above two sets of initial conditions are very large even though these two sets of initial conditions are differed only by $1 \%$. This implies that the system is very sensitive to the initial condition. To investigate the sensitivity of the system parameters against the system dynamics, since there are six system parameters in the HIV model, namely, $a, b$, $c, d, e$ and $f$, the range of the concentrations of the CD4 lymphocyte population, CD8 lymphocyte population and the HIV-1 viral load are plotted with respect to these system parameters. Figure 4a, Figure $4 \mathrm{~b}$ and Figure $4 \mathrm{c}$ plot the ranges of $x(t), y(t)$ and $z(t)$ as $a$ varies when $b=50, c=0.25$, $d=10, e=0.01, f=0.006, x_{0}=1000, y_{0}=550, x(0)=x_{0}, y(0)=y_{0}$ and $z(0)=0.03$. Similarly, Figure 5 to Figure 9 plot the corresponding ranges as the parameter $b$ to the parameter $f$ vary, respectively. From these computer numerical simulation results, we can see that the parameters $d$ and $e$ are the most sensitive to the system dynamics. Hence, we can conclude that the system is chaotic and exhibits a bifurcation phenomenon. This chaotic nature of the system causes the formulation of the control strategy being very challenging.

\section{PROPOSED CONTROL STRATEGY}

Denote $U(t)$ as the dose concentration. This corresponds to the control force of the system. Assume that $U(t)$ is applying directly to $z(t)$, that is:

$$
\begin{aligned}
& \dot{x}(t)=a\left(x_{0}-x(t)\right)-b x(t) z(t), \\
& \dot{y}(t)=c\left(y_{0}-y(t)\right)+d y(t) z(t)
\end{aligned}
$$

and

$$
\dot{z}(t)=z(t)(e x(t)-f y(t))-U(t)
$$

Consider the following control force

$$
U(t)=\frac{e^{2}(x(t))^{2} z(t)}{4 f y(t)}
$$

and define 
International Journal of Bifurcation and Chaos

$$
V(t)=(z(t))^{2}
$$

It can be easily seen that $V(t)>0$ for $z(t) \neq 0$, and

$$
\dot{z}(t)=-\frac{z(t)(e x(t)-2 f y(t))^{2}}{4 f y(t)} .
$$

Consequently, we have

$$
\dot{V}(t)=-\frac{(z(t))^{2}(e x(t)-2 f y(t))^{2}}{2 f y(t)} .
$$

As

$$
\dot{x}(t)=a\left(x_{0}-x(t)\right)-b x(t) z(t)
$$

we have

$$
x(t)=\frac{\int_{-\infty}^{t} a x_{0} \exp \left(\int_{-\infty}^{\xi}(a+b z(\tau)) d \tau\right) d \xi}{\exp \left(\int_{-\infty}^{t}(a+b z(\tau)) d \tau\right)} .
$$

Similarly, as

$$
\dot{y}(t)=c\left(y_{0}-y(t)\right)+d y(t) z(t)
$$

we have

$$
y(t)=\frac{\int_{-\infty}^{t} c y_{0} \exp \left(\int_{-\infty}^{\xi}(c-d z(\tau)) d \tau\right) d \xi}{\exp \left(\int_{-\infty}^{t}(c-d z(\tau)) d \tau\right)} .
$$

Since $a, c, x_{0}$ and $y_{0}$ are positive, from (9) and (11), we can conclude that $x(t)>0$ and $y(t)>0$ $\forall t \geq 0$. By putting $y(t)>0$ and $f>0$ to (7), we can conclude that $\dot{V}(t)<0 \quad \forall t \geq 0$. Since we assume that $z(0)>0$, we have $\dot{z}(t)<0 \forall t \geq 0, z(t)>0 \forall t \geq 0$ and $\lim _{t \rightarrow+\infty} z(t)=0$. By putting $f>0$, $y(t)>0$ and $z(t)>0$ to (4), we have $U(t)>0 \forall t \geq 0$. When $z(t) \approx 0 \forall t \geq T$, where $T$ is a positive real number, from (9) and (11), we have

$$
x(t) \approx x_{0}+\left(x(T)-x_{0}\right) \exp (a(T-t))
$$

and

$$
y(t) \approx y_{0}+\left(y(T)-y_{0}\right) \exp (c(T-t)) \forall t \geq T
$$


International Journal of Bifurcation and Chaos

Since $a$ and $c$ are positive, we have

$$
\lim _{t \rightarrow+\infty} x(t) \approx x_{0}
$$

and

$$
\lim _{t \rightarrow+\infty} y(t) \approx y_{0}
$$

Consequently, both $x(t)$ and $y(t)$ are bounded and converge to the corresponding equilibrium values. Hence, this control strategy can guarantee the asymptotical stability of the system, the monotonic decrement of the concentration of the HIV-1 viral load, as well as the satisfaction of the positivity constraint of the system states and that of the dose concentration.

Although this control strategy guarantees that the system states will converge to the equilibrium point, the convergent rate is slow because $V(t)$ is independent of both $x(t)$ and $y(t)$. Now, consider another control force as follows:

$$
U(t)=\frac{e^{2}(x(t))^{2} z(t)}{4 f y(t)}+\frac{k_{2} d}{k_{3}}\left(y(t)-\frac{y_{0}}{2}\right)^{2}+\frac{k_{1} b x_{0}^{2}}{4 k_{3}}
$$

and define

$$
V(t)=k_{1}\left(x_{0}-x(t)\right)^{2}+k_{2}\left(y_{0}-y(t)\right)^{2}+k_{3}(z(t))^{2}
$$

where $k_{1}$ and $k_{2}$ are non-negative constants as well as $k_{3}$ is a positive constant. Obviously, the previous control strategy is a particular case of this control strategy. Now, we have

$$
\dot{z}(t)=-\frac{z(t)(e x(t)-2 f y(t))^{2}}{4 f y(t)}-\frac{k_{2} d}{k_{3}}\left(y(t)-\frac{y_{0}}{2}\right)^{2}-\frac{k_{1} b x_{0}^{2}}{4 k_{3}}
$$

and

$$
\dot{V}(t)=-\frac{k_{3}(z(t))^{2}(e x(t)-2 f y(t))^{2}}{2 f y(t)}-2 k_{2} c\left(y_{0}-y(t)\right)^{2}-2 k_{1} a\left(x_{0}-x(t)\right)^{2}-2 k_{1} b z(t)\left(x(t)-\frac{x_{0}}{2}\right)^{2}-\frac{k_{2} d z(t) y_{0}^{2}}{2} .(19
$$

It can be checked easily that $V(t)>0$ for $(x(t), y(t), z(t)) \neq\left(x_{0}, y_{0}, 0\right)$. Similarly, from (9) and (11), we have $x(t)>0$ and $y(t)>0 \quad \forall t \geq 0$. If $z(t)>0 \quad \forall t \geq 0$, then $U(t)>0, \dot{V}(t)<0$ and $\dot{z}(t)<0 \quad \forall t \geq 0$. Hence, the success of this control strategy highly depends whether $z(t)>0 \quad \forall t \geq 0$ or not. However, in general it is not guaranteed that $z(t)>0 \quad \forall t \geq 0$ is satisfied even though this control strategy could achieve a fast convergent rate because $V(t)$ depends on both $x(t)$ and $y(t)$. 
International Journal of Bifurcation and Chaos

Now, consider an uncontrolled case, that is:

$$
U(t)=0
$$

Define

$$
V(t)=(z(t))^{2}
$$

then we have

$$
\dot{z}(t)=z(t)(e x(t)-f y(t))
$$

and

$$
\dot{V}(t)=2(z(t))^{2}(e x(t)-f y(t))
$$

It can be checked easily that $V(t)>0$ for $z(t) \neq 0$. Similarly, from (9) and (11), we have $x(t)>0$ and $y(t)>0 \forall t \geq 0$. If $e x(t)-f y(t)<0$, then $\dot{V}(t)<0$. Since we assume that $z(0)>0$, we have $\dot{z}(t)<0$ $\forall t \geq 0, z(t)>0 \forall t \geq 0$ and $\lim _{t \rightarrow+\infty} z(t)=0$. Similarly, we have $\lim _{t \rightarrow+\infty} x(t) \approx x_{0}$ and $\lim _{t \rightarrow+\infty} y(t) \approx y_{0}$. Hence, the success of the uncontrolled strategy highly depends whether $e x(t)-f y(t)<0$ or not. However, in general it is not guaranteed that $e x(t)-f y(t)<0$ is satisfied even though the uncontrolled strategy does not require patients taking medications and patients do not need to tolerate the side effects of the medications.

Now, consider the following control strategy:

\section{Theorem 1}

Assume that $a, b, c, d, e, f, x_{0}, y_{0}, k_{1}, k_{2}, k_{3}, x(0), y(0), z(0)>0$. Denote $\alpha$ as a positive constant.

Case I: If $z(t)>\alpha$, then

$$
U(t)=\frac{e^{2}(x(t))^{2} z(t)}{4 f y(t)}+\frac{k_{2} d}{k_{3}}\left(y(t)-\frac{y_{0}}{2}\right)^{2}+\frac{k_{1} b x_{0}^{2}}{4 k_{3}} .
$$

Case II: If $\alpha \geq z(t)>0$ and $e x(t)-f y(t) \geq 0$, then

$$
U(t)=\frac{e^{2}(x(t))^{2} z(t)}{4 f y(t)} .
$$

Case III: If $\alpha \geq z(t)>0$ and $e x(t)-f y(t)<0$, then

$$
U(t)=0 \text {. }
$$


The overall controlled system is asymptotically stable, the concentration of the HIV-1 viral load is monotonic decreasing, as well as the positivity constraint of the system states and that of the dose concentration is guaranteed to be satisfied.

Proof:

Since $\dot{z}(t)<0$ for all these three cases and $z(0)>0$, the system can only switch from Case I to either Case II or Case III, but not vice versa. If $z(0) \leq \alpha$, then the system can only operate in Case II and Case III. If $z(0)>\alpha$, as $\dot{z}(t)<0, z(t)$ is decreasing until it reaches the value $\alpha$ and then switches to either Case II or Case III. Hence, the objective of the control strategy defined by Case I is to accelerate the transient response. Once the system is operating under the control strategy defined by either Case II or Case III, as $\dot{V}(t)<0, \dot{z}(t)<0, x(t)>0, y(t)>0, z(t)>0$ and $U(t) \geq 0 \forall t \geq 0$ as well as $\lim _{t \rightarrow+\infty} x(t) \approx x_{0}, \lim _{t \rightarrow+\infty} y(t) \approx y_{0}$ and $\lim _{t \rightarrow+\infty} z(t)=0$ for both Case II and Case III, the positivity constraint of the system states and that of the dose concentration will be satisfied, the concentration of the HIV-1 viral load will be monotonic decreasing, as well as the asymptotical stability of the overall system will be guaranteed. This completes the proof.

There are four parameters in the controlled system, namely, $\alpha, k_{1}, k_{2}$ and $k_{3}$. Actually Theorem 1 is valid $\forall \alpha, k_{1}, k_{2}, k_{3}>0$. To design the value of $\alpha$, since $\alpha$ is the concentration of the HIV-1 viral load in which the control strategy is switched from Case I to either Case II or Case III, and the rate of the change of the concentration of the HIV-1 viral load under the control strategy defined by Case I is faster than that defined by Case II and Case III, $\alpha$ should be small so that the concentration of the HIV-1 viral load will decrease at a faster rate. However, as $\dot{z}(t) \neq 0$ when $z(t)=0$, too small value of $\alpha$ would cause a rapid change of the state trajectory near the equilibrium point. Consequently, patients cannot stop the therapy. To obtain a balance between these two factors, we choose $\alpha$ to be a value equal to $59.5140 \%$ of $z(0)$ in our computer numerical simulation, that is 0.0179 . For the values of $k_{1}, k_{2}$ and $k_{3}$, from (16), we see that $U(t)$ is small if $k_{1}$ and $k_{2}$ are small and $k_{3}$ is large. In the practical situation, $U(t)$ should be small so that the side effects of the 
International Journal of Bifurcation and Chaos

medications are negligible. However, we can see from (18) that the rate of change of the concentration of the HIV-1 viral load is large if $k_{1}$ and $k_{2}$ are large and $k_{3}$ is small. As a fast response is preferred, there is a tradeoff between the strength of the side effects and the convergent rate of the system. To obtain a balance between these two factors, we propose to solve the problem via an optimization approach as follows:

For the control strategy defined by Case I, since

$$
\dot{U}(t)=\dot{y}(t)\left[\frac{2 k_{2} d}{k_{3}}\left(y(t)-\frac{y_{0}}{2}\right)-\frac{e^{2}(x(t))^{2} z(t)}{4 f(y(t))^{2}}\right]+\frac{e^{2}}{4 f}\left(\frac{2 x(t) z(t) \dot{x}(t)+(x(t))^{2} \dot{z}(t)}{y(t)}\right)
$$

and $\dot{z}(t)<0 \quad \forall t \geq 0$, if $\dot{x}(t)<0, \dot{y}(t)>0$ and

$$
\frac{2 k_{2} d}{k_{3}}\left(y(t)-\frac{y_{0}}{2}\right)<\frac{e^{2}(x(t))^{2} z(t)}{4 f(y(t))^{2}} \forall z(t)>\alpha,
$$

then we have $\dot{U}(t)<0 \quad \forall z(t)>\alpha$. This implies that the maximum value of $U(t)$ will occur at $t=0$ if the above conditions are satisfied. Define an acceptable bound on the dose concentration as $U_{\max }$. Then $k_{1}, k_{2}$ and $k_{3}$ are designed such that $U(t)<U_{\max } \forall z(t)>\alpha$. That is:

$$
\frac{e^{2}(x(0))^{2} z(0)}{4 f y(0)}+\frac{k_{2} d}{k_{3}}\left(y(0)-\frac{y_{0}}{2}\right)^{2}+\frac{k_{1} b x_{0}^{2}}{4 k_{3}}<U_{\max } .
$$

Similarly, we define an acceptable bound on the rate of change of the concentration of the HIV-1 viral load and on the rate of change of $V(t)$ at $t=0$ as $\dot{z}_{\max }$ and $\dot{V}_{\max }$, respectively. From (18) and (19), we have

$$
-\frac{z(0)}{4 f y(0)}(e x(0)-2 f y(0))^{2}-\frac{k_{2} d}{k_{3}}\left(y(0)-\frac{y_{0}}{2}\right)^{2}-\frac{k_{1} b x_{0}^{2}}{4 k_{3}}<\dot{z}_{\max }
$$

and

$-\frac{k_{3}(z(0))^{2}}{2 f y(0)}(e x(0)-2 f y(0))^{2}-2 k_{2} c\left(y_{0}-y(0)\right)^{2}-2 k_{1} a\left(x_{0}-x(0)\right)^{2}-2 k_{1} b z(0)\left(x(0)-\frac{x_{0}}{2}\right)^{2}-\frac{k_{2} d z(0) y_{0}^{2}}{2}<\dot{V}_{\max }$

(25)-(27), as well as $k_{1}, k_{2}$ and $k_{3}$ being positive, forms a linear matrix inequality constraint on $k_{1}$, $k_{2}$ and $k_{3}$. For most of patients, $U_{\max }$ should be set around 0.37 . Hence, we choose $U_{\max }$ accordingly. In order to have a fast response, $\dot{z}_{\max }$ and $\dot{V}_{\max }$ should be as small as possible, hence we choose $\dot{z}_{\max }$ 
and $\dot{V}_{\max }$ as -0.15 and -0.0095 , respectively.

Due to the tradeoff between the strength of the side effects and the convergent rate of the system, we formulate the following optimization problem as follows:

\section{Problem $(\mathbf{P})$}

$$
\begin{array}{ll}
\min _{\left(k_{1}, k_{2}, k_{3}\right)} & k_{1}+k_{2}+k_{3}, \\
\text { subject to } & (25)-(27) \\
\text { and } & k_{1}, k_{2} \text { and } k_{3} \text { are positive. }
\end{array}
$$

Problem $\mathbf{P}$ is a standard linear programming problem, many existing solvers, such as Matlab optimization toolbox, can be employed for solving the problem. Moreover, as this optimization problem is convex, the obtained solution will be globally optimal if the feasible set is nonempty.

Figure 10 plots the controlled state responses with the system parameters and the initial condition being the same as those applied in Figure 1, where $\alpha, k_{1}, k_{2}$ and $k_{3}$ are determined based on the above discussion. It can be seen from the figure that the concentration of the HIV-1 viral load is monotonic decreasing, the system states asymptotically converge to the equilibrium point, as well as the concentrations of the CD4 lymphocyte population, the CD8 lymphocyte population and the HIV-1 viral load are non-negative and bounded. Hence, the proposed control strategy satisfies the positivity constraint. Figure 11 shows the corresponding dose concentration. It can be seen from the figure that the maximum dose concentration within the first 4 weeks is 0.37 , which satisfies the specification. The dose concentration is monotonic decreasing within the first 4 weeks, but it drops dramatically to low values after the 4th week because the system switches from the control strategy defined by Case I to that defined by Case II. Between the 85.7 th and 219.7 th weeks, the dose concentration is exactly equal to zero. This implies that the system switches to the control strategy defined by Case III and no therapy is required. After the 219.7th week, the system switches back to the control strategy defined by Case II, so the dose concentration is non-zero and maintains at low level. To illustrate the effectiveness of the control strategy defined by Case I, Figure 12 plots the controlled state responses 
International Journal of Bifurcation and Chaos

of the system switched only between Case II and Case III. Figure 13 plots the corresponding dose concentration with the system parameters and the initial condition being the same as those applied in Figure 1. It can be seen from the figure that the concentration of the HIV-1 viral load decreases slowly while that based on the control strategy defined by Case I drops to the value equal to $\alpha$ quickly (at the 4th week).

\section{CONCLUSIONS}

In this paper, we suggest to initiate the therapy based on both the concentration of the HIV-1 viral load and the ratio of the CD4 lymphocyte population to the CD8 lymphocyte population. If the concentration of the HIV-1 viral load is higher than a threshold, then the first type of therapy will be applied. If the concentration of the HIV-1 viral load is lower than or equal to the threshold and the ratio of the $\mathrm{CD} 4$ lymphocyte population to the $\mathrm{CD} 8$ lymphocyte population is greater than another threshold, then the second type of therapy will be applied. Otherwise, no therapy will be applied. The advantages of the proposed control strategy are that the therapy can be stopped under certain conditions, while the state variables of the overall system is asymptotically stable with fast convergent rate, the concentration of the controlled HIV-1 viral load is monotonic decreasing, as well as the positivity constraint of the system states and that of the dose concentration is guaranteed to be satisfied.

\section{ACKNOWLEDGEMENT}

The work obtained in this paper was supported by a research grant from Queen Mary, University of London.

\section{REFERENCES}

[1] Fernando Menezes Campello de Souza, "Modeling the dynamics of HIV-1 and CD4 and CD8 lymphocytes," IEEE Engineering in Medicine and Biology, vol. 18, no. 1, pp. 21-24, 1999. 
International Journal of Bifurcation and Chaos

[2] Michael E. Brandt and Guanrong Chen, "Feedback control of a biodynamical model of HIV-1," IEEE Transactions on Biomedical Engineering, vol. 48, no. 7, pp. 754-759, 2001.

[3] Ji Hyun Ko, Won Hee Kim and Chung Choo Chung, "Optimized structured treatment interruption for HIV therapy and its performance analysis on controllability," IEEE Transactions on Biomedical Engineering, vol. 53, no. 3, pp. 380-386, 2006.

[4] Ian K. Craig and Xiaohua Xia, "Can HIV/AIDS be controlled? Applying control engineering concepts outside traditional fields," IEEE Control Systems Magazine, vol. 25, no. 1, pp. 80-83, 2005 .

[5] Shuzhi Sam Ge, Zhiling Tian and Tong Heng Lee, "Nonlinear control of a dynamic model of HIV-1," IEEE Transactions on Biomedical Engineering, vol. 52, no. 3, pp. 353-361, 2005.

[6] Dhiraj Arora, Mikhail Skliar and Robert B. Roemer, "Minimum-time thermal dose control of thermal therapies," IEEE Transactions on Biomedical Engineering, vol. 52, no. 2, pp. 191-200, 2005.

[7] Annah M. Jeffrey, Xiaohua Xia and Ian K. Craig, "When to initiate HIV therapy: a control theoretic approach," IEEE Transactions on Biomedical Engineering, vol. 50, no. 11, pp. 1213-1220, 2003.

[8] Ian K. Craig, Xiaohua Xia and Juliana W. Venter, "Introducing HIV/AIDS education into the electrical engineering curriculum at the university of Pretoria," IEEE Transactions on Education, vol. 47, no. 1, pp. 65-73, 2004.

[9] R.A. Filter, X. Xia and C. M. Gray, "Dynamic HIV/AIDS parameter estimation with application to a vaccine readiness study in Southern Africa," IEEE Transactions on Biomedical Engineering, vol. 52, no. 5, pp. 784-791, 2005.

[10] X. Xia and C. H. Moog, "Identifiability of nonlinear systems with application to HIV/AIDS models," IEEE Transactions on Automatic Control, vol. 48, no. 2, pp. 330-336, 2003.

[11] B. J. Hammond, "Quantitative study of the control of HIV-1 gene expression," Journal of Theoretical Biology, vol. 163, pp. 199-221, 1993. 
International Journal of Bifurcation and Chaos

[12] Lawrence M. Wein, Stefanos A. Zenios and Martin A. Nowak, "Dynamic multidrug therapies for HIV: a control theoretic approach," Journal of Theoretical Biology, vol. 185, pp. 15-29, 1997.

[13] Rob J. de Boer and Alan S. Perelson, "Target cell limited and immune control models of HIV infection: a compression,” Journal of Theoretical Biology, vol. 190, pp. 201-214, 1998.

[14] Lawrence M. Wein, Rebecca M. D’Amato and Alan S. Perelson, "Mathematical analysis of antiretroviral therapy aimed at HIV-1 eradication or maintenance of low viral loads," Journal of Theoretical Biology, vol. 192, pp. 81-98, 1998.

[15] Narendra M. Dixit and Alan S. Perelson, "Complex patterns of viral load decay under antiretroviral therapy: influence of pharmacokinetics and intracellular delay," Journal of Theoretical Biology, vol. 226, pp. 95-109, 2004.

[16] Max A. Strfford, Lawerence Corey, Yunzhen Cao, Eric S. Daar, David D. Ho and Alan S. Perelson, "Modeling plasma virus concentration during primary HIV infection," Journal of Theoretical Biology, vol. 203, pp. 285-301, 2000.

[17] Michael A. Fishman and Alan S. Perelson, "Th1/Th2 cross regulation," Journal of Theoretical Biology, vol. 170, pp. 25-56, 1994.

[18] Paulina Essunger and Alan S. Perelson, "Modeling HIV infection of CD4 ${ }^{+}$T-cell subpopulations,” Journal of Theoretical Biology, vol. 170, pp. 367-391, 1994.

[19] Richard M. Berry and Martin A. Nowak, "Defective escape mutants of HIV," Journal of Theoretical Biology, vol. 171, pp. 387-395, 1994.

[20] Marc Lipsitch and Martin A. Nowak, "The evolution of virulence in sexually transmitted HIV/AIDS,” Journal of Theoretical Biology, vol. 174, pp. 427-440, 1995.

[21] Martin A. Nowak, Robert M. May and Karl Sigmund, "Immune responses against multiple epitopes," Journal of Theoretical Biology, vol. 175, pp. 352-353, 1995.

[22] Franck Courchamp, Dominique Pontier, Michel Langlais and Marc Artois, "Population dynamics of feline immunodeficiency virus within cat populations," Journal of Theoretical Biology, vol. 175, pp. 553-560, 1995. 
International Journal of Bifurcation and Chaos

[23] Yoh Iwasa, Franziska Michor and Martin A. Nowak, "Virus evolution within patients increases pathogenicity,” Journal of Theoretical Biology, vol. 232, pp. 17-26, 2005.

[24] Martin A. Nowak, Sebastian Bonhoeffer, George M. Shaw and Robert M. May, “Anti-viral drug treatment: dynamics of resistance in free virus and infected cell populations," Journal of Theoretical Biology, vol. 184, pp. 203-217, 1997.

[25] Dominik Wodarz, Alun L. Lloyd, Vincent A. A. Jansen and Martin A. Nowak, "Dynamics of macrophage and T cell infection by HIV," Journal of Theoretical Biology, vol. 196, pp. 101-113, 1999.

[26] Michael A. Gilchrist, Daniel Coombs and Alan S. Perelson, "Optimizing within-host viral fitness: infected cell lifespan and virion production rate," Journal of Theoretical Biology, vol. 229, pp. 281-288, 2004.

[27] R. M. Jafelice, L. C. Barros, R. C. Bassanezi F. Gomide, "Fuzzy set-based model to compute the life expectancy of HIV infected populations," IEEE Annual Meeting of the Fuzzy Information Processing, NAFIPS, vol. 1, pp. 314-318, 27-30 June 2004.

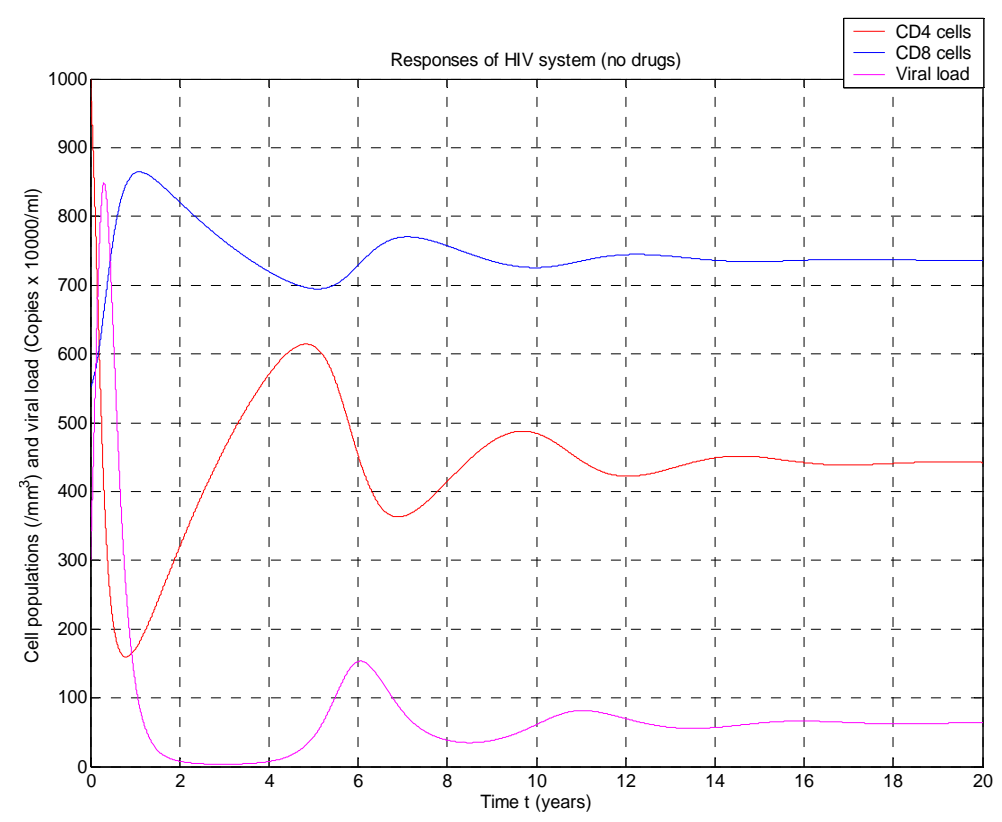

Figure 1a. State responses of the system. 


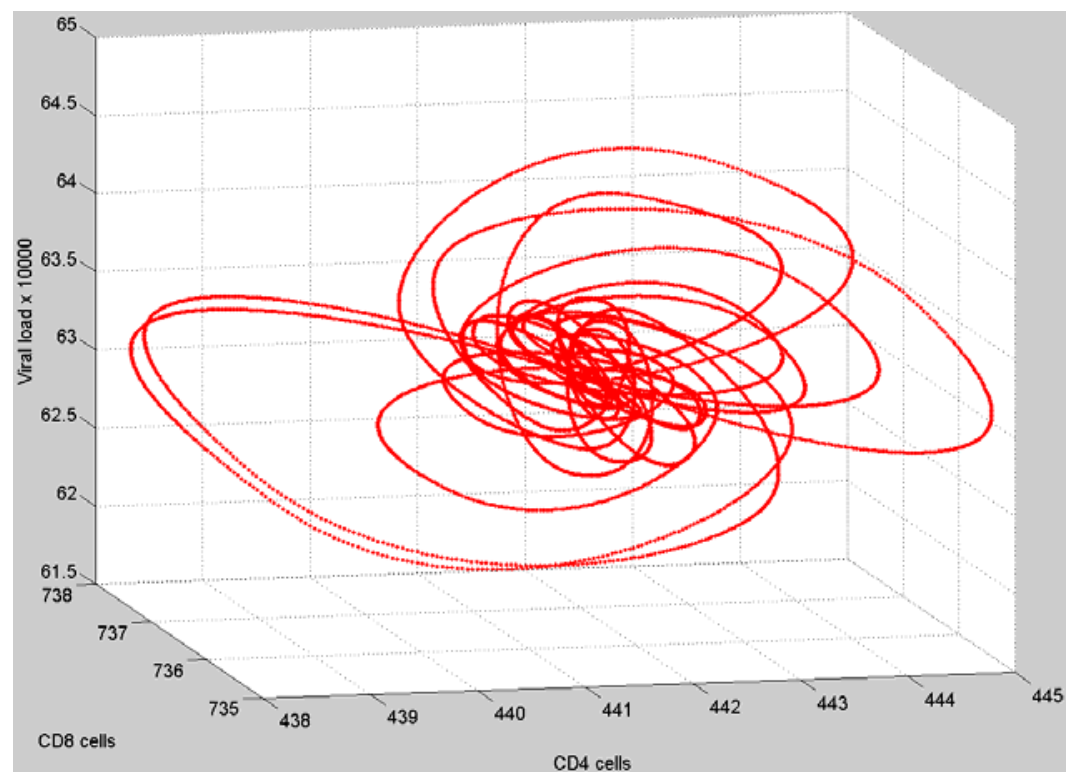

Figure $1 \mathrm{~b}$. The state trajectory of the system.

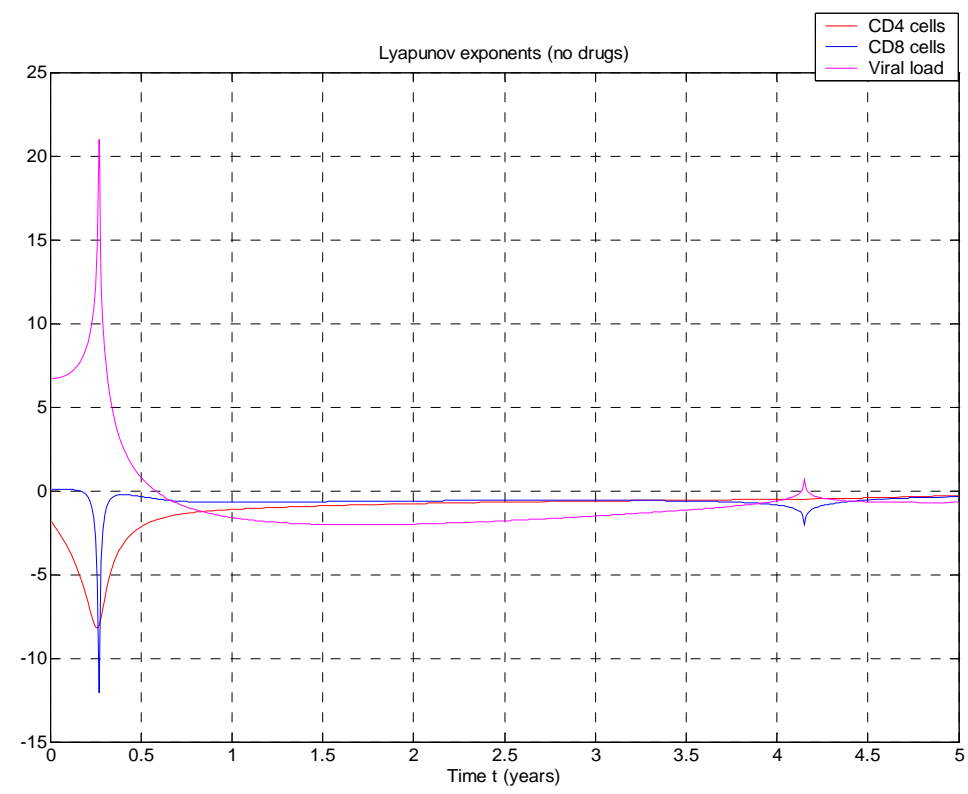

Figure 2. Lyapunov exponents of the system. 


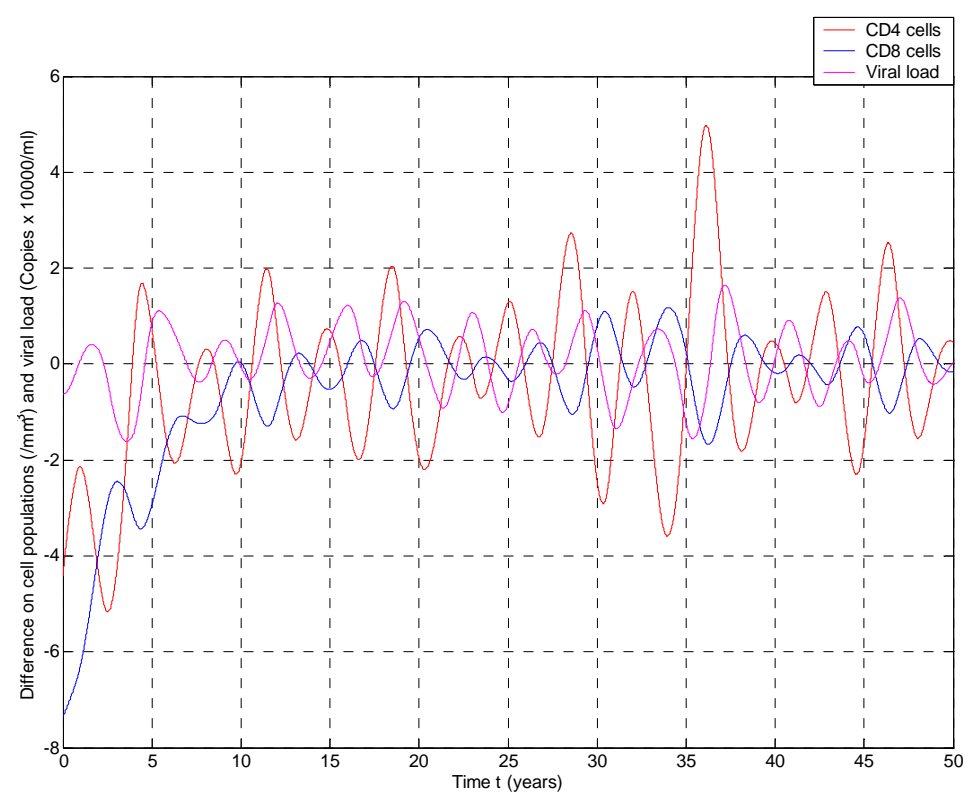

Figure 3. Difference of the responses of the CD4 lymphocyte population, the CD8 lymphocyte population and the HIV-1 viral load at different initial conditions.
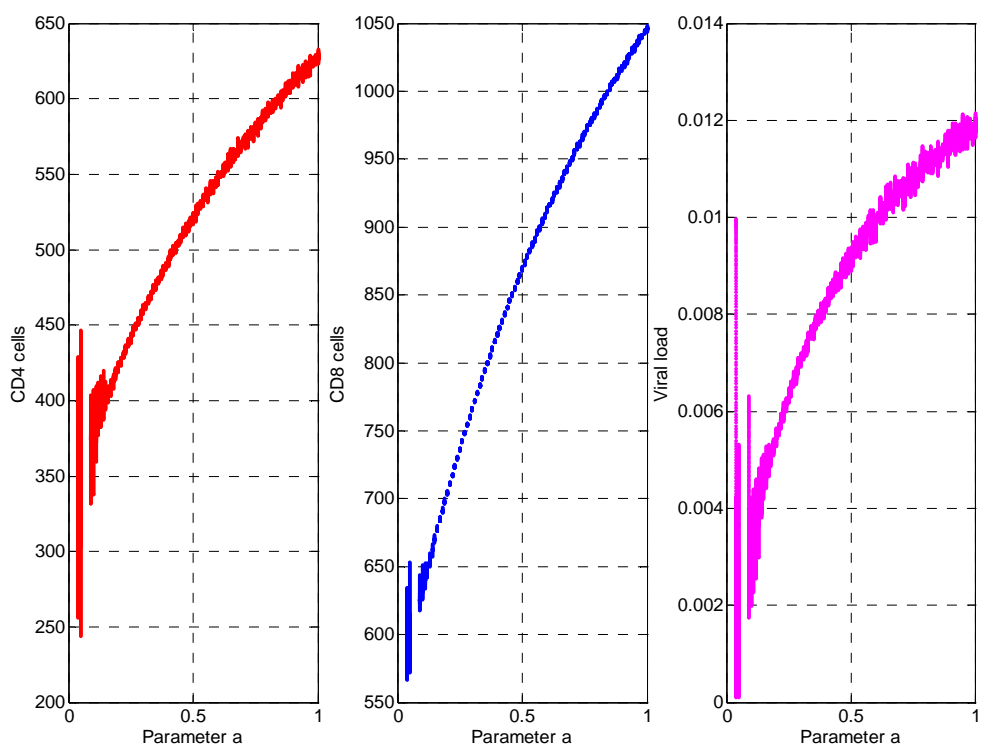

Figure 4. Ranges of the CD4 lymphocyte population, the CD8 lymphocyte population and the HIV-1 viral load as parameter $a$ varies. 
International Journal of Bifurcation and Chaos
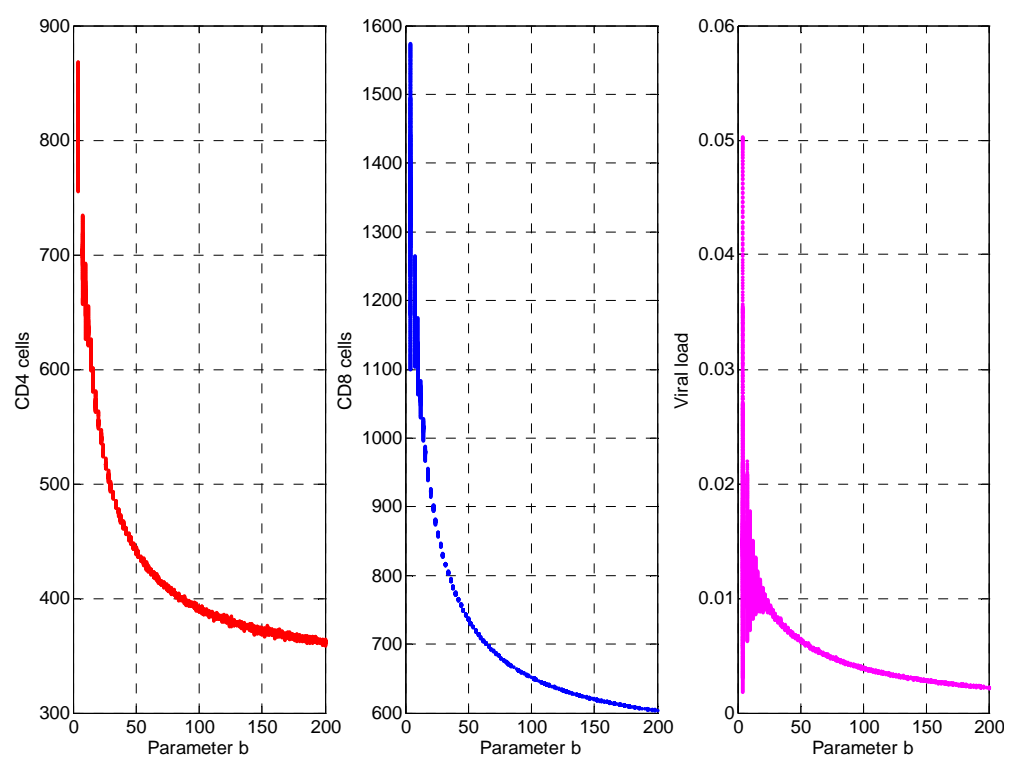

Figure 5. Ranges of the CD4 lymphocyte population, the CD8 lymphocyte population and the HIV-1 viral load as parameter $b$ varies.
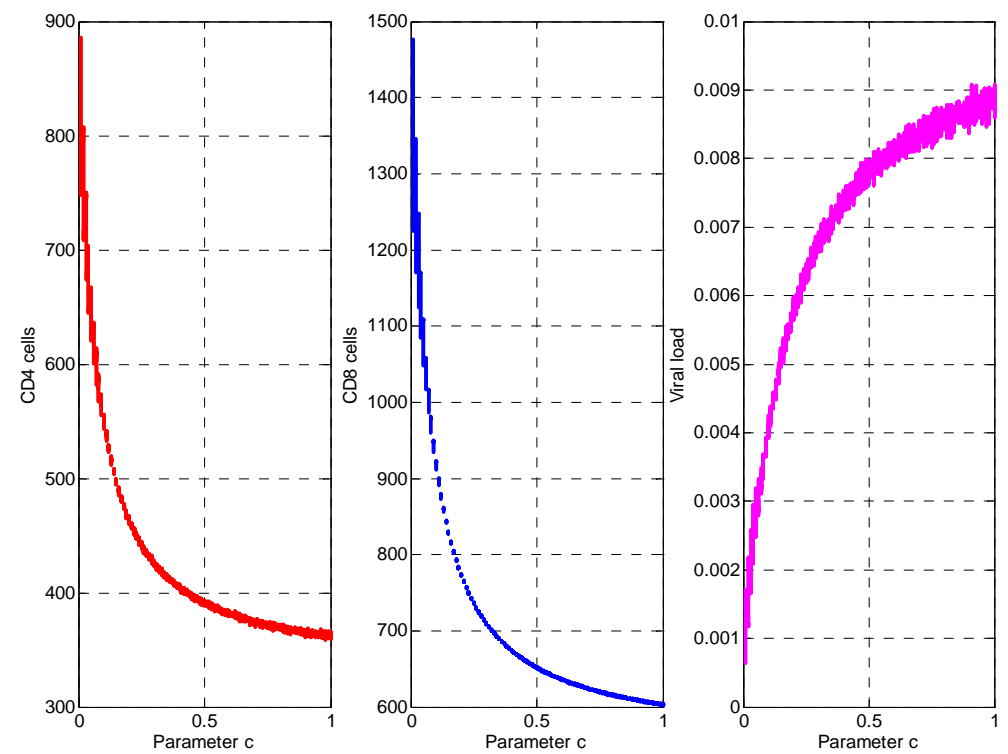

Figure 6. Ranges of the CD4 lymphocyte population, the CD8 lymphocyte population and the HIV-1 viral load as parameter $c$ varies. 
International Journal of Bifurcation and Chaos
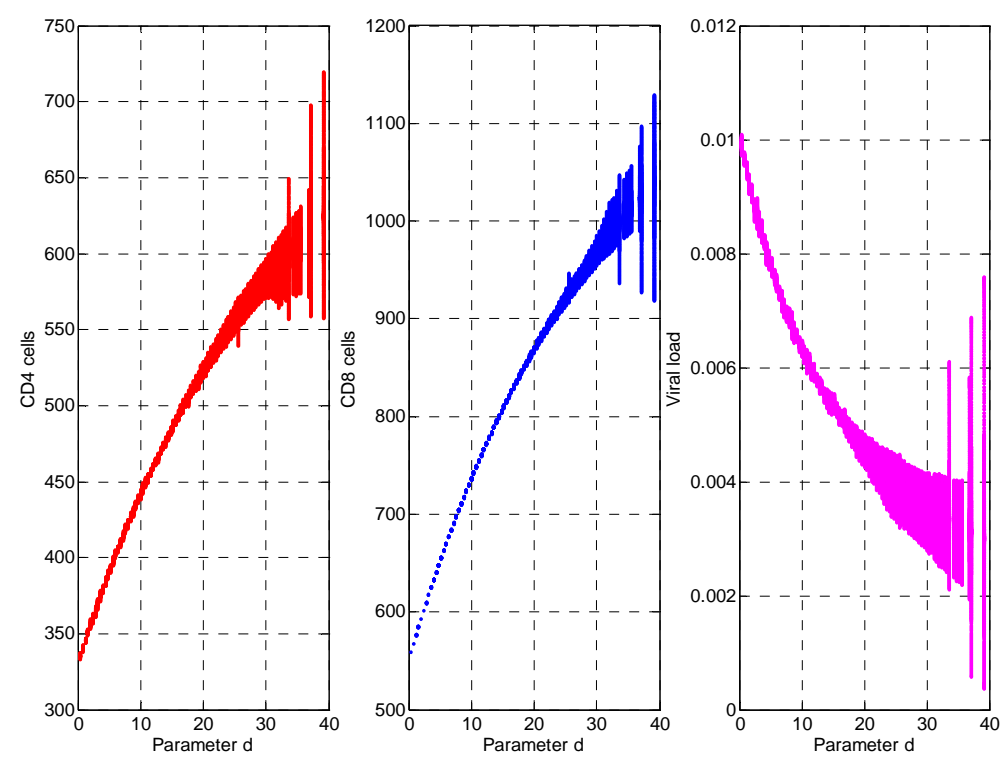

Figure 7. Ranges of the CD4 lymphocyte population, the CD8 lymphocyte population and the HIV-1 viral load as parameter $d$ varies.
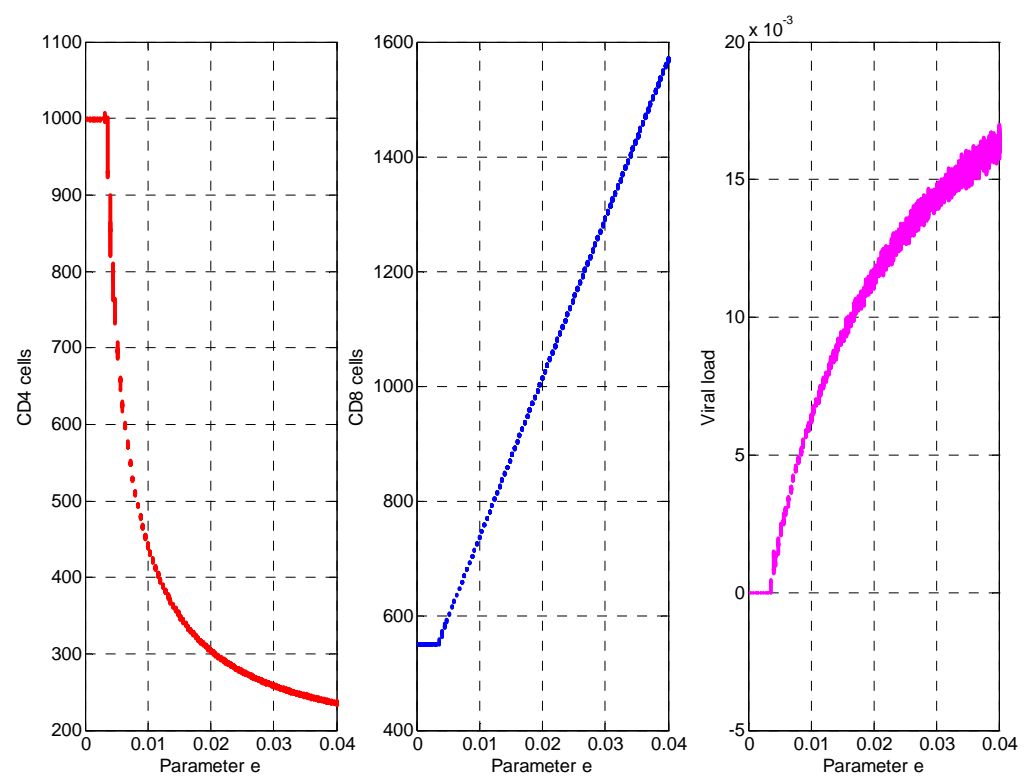

Figure 8. Ranges of the CD4 lymphocyte population, the CD8 lymphocyte population and the HIV-1 viral load as parameter $e$ varies. 
International Journal of Bifurcation and Chaos
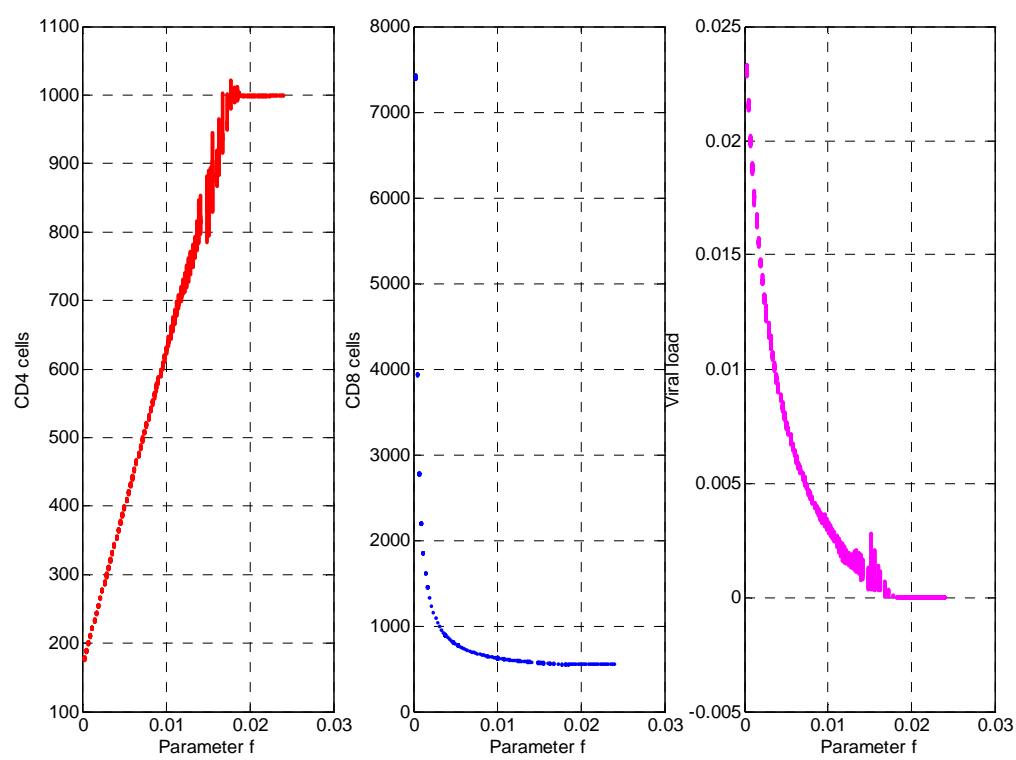

Figure 9. Ranges of the CD4 lymphocyte population, the CD8 lymphocyte population and the HIV-1 viral load as parameter $f$ varies.

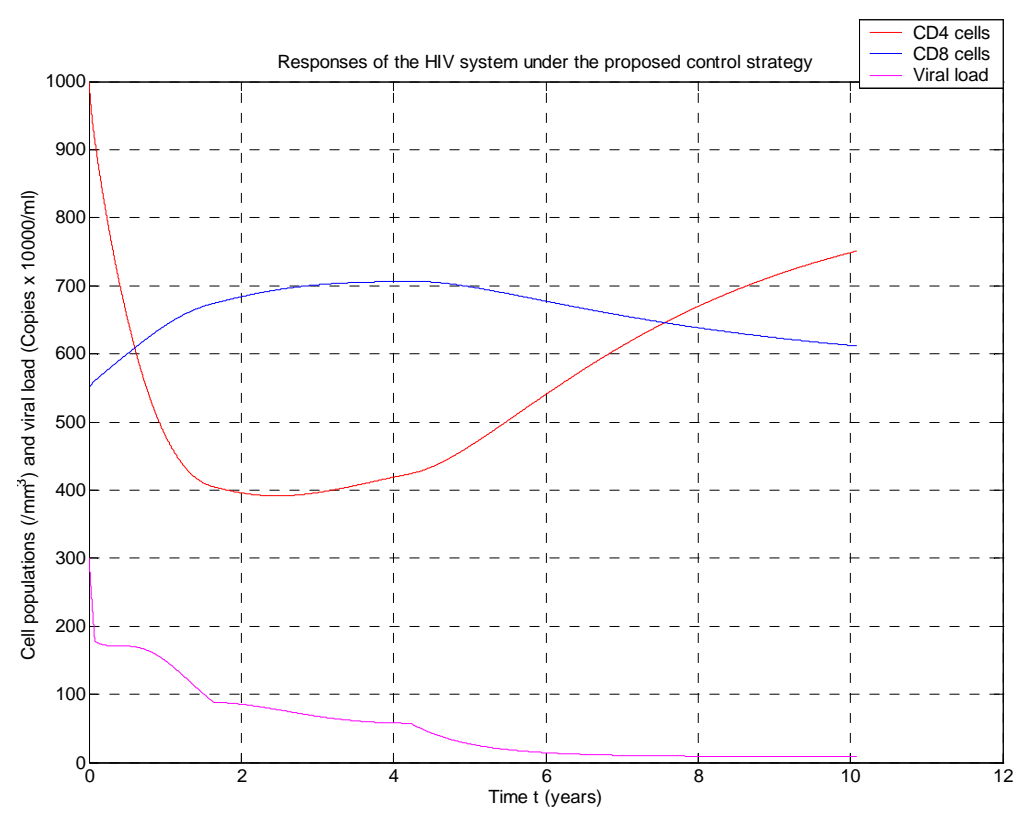

Figure 10. State responses of the overall controlled system. 
International Journal of Bifurcation and Chaos

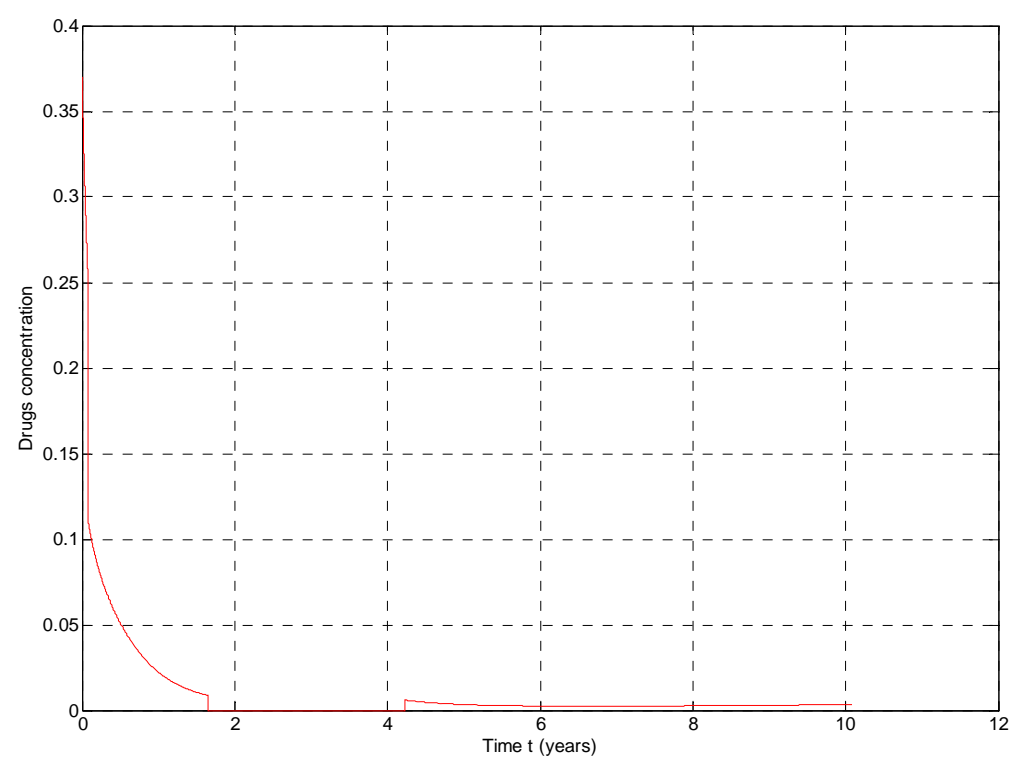

Figure 11. Dose concentration.

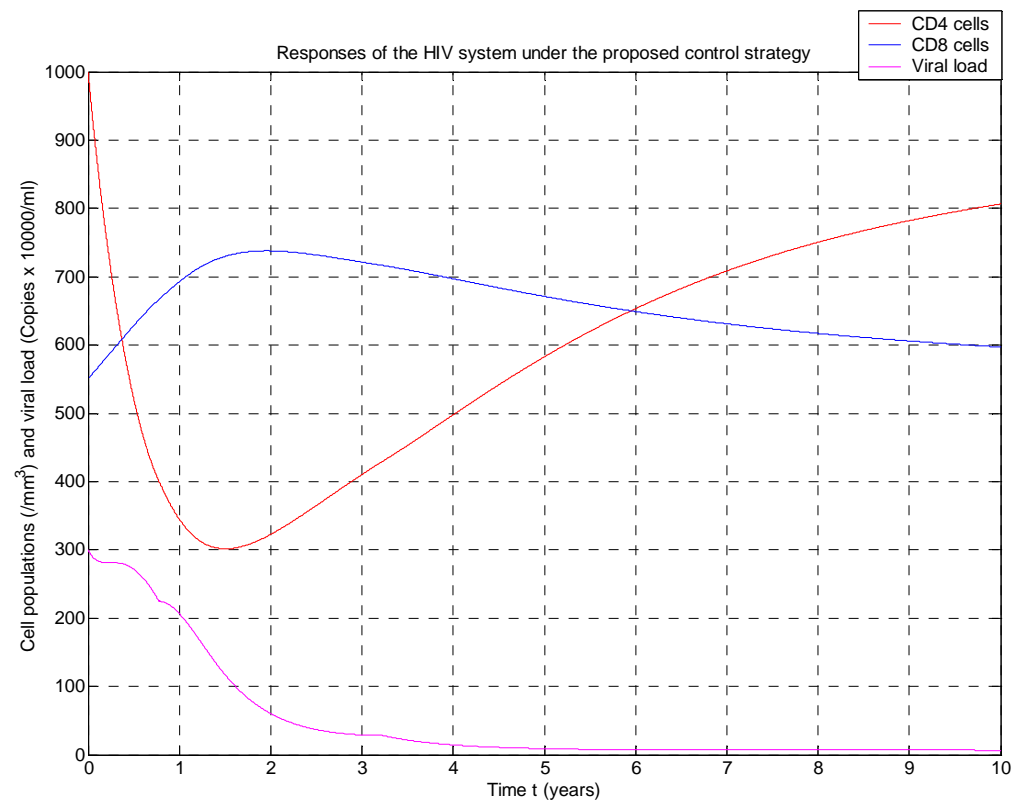

Figure 12. State responses of the overall controlled system. 
International Journal of Bifurcation and Chaos

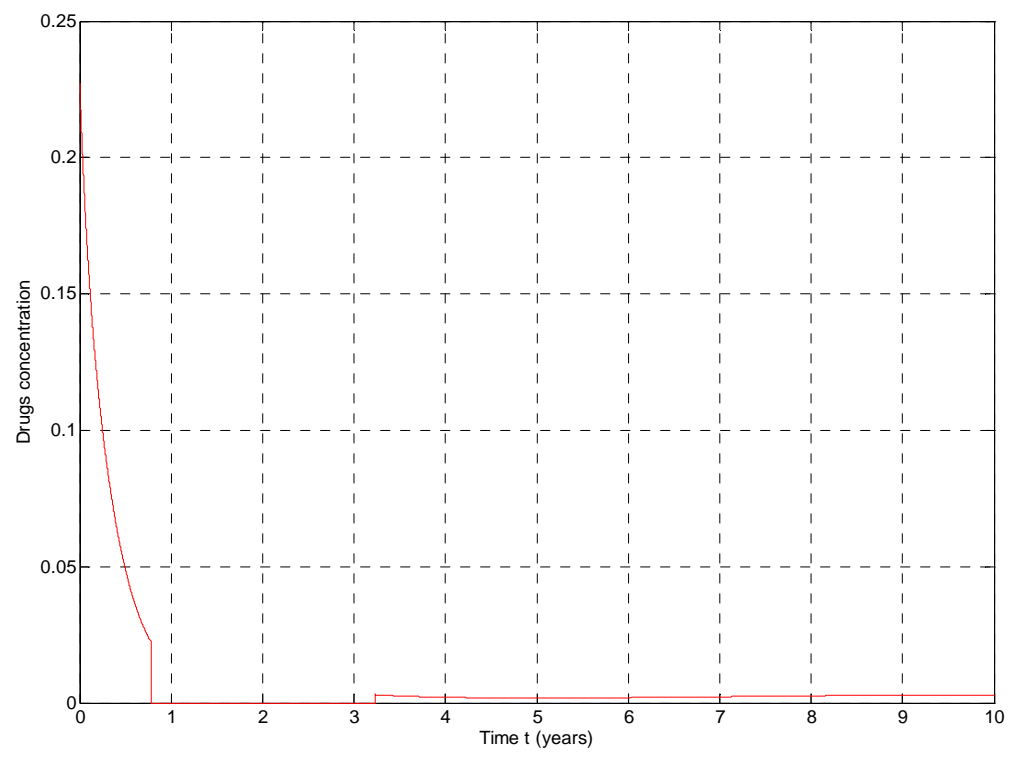

Figure 13. Dose concentration. 